\title{
Socioeconomic inequalities in health: Measurement, computation, and statistical inference
}

\author{
Nanak Kakwani*a ${ }^{*}$, Adam Wagstaff ${ }^{\mathrm{b}}$, Eddy van Doorslaer \\ "School of Economics, University of New' South Wales, Sydney', NSW 2052, Australia \\ ${ }^{b}$ School of Social Sciences, University of Sussex, Brighton BNI 9QN, UK \\ 'Department of Health Policy and Management. Erasmus University. 3000 DR Rotterdam. \\ The Netherlands
}

\begin{abstract}
This paper clarifies the relationship between two widely used indices of health inequality and explains why these are superior to others indices used in the literature. It also develops asymptotic estimators for their variances and clarifies the role that demographic standardization plays in the analysis of socioeconomic inequalities in health. Empirical illustrations are presented for Dutch health survey data.
\end{abstract}

Key' words: Socioeconomic inequality; Concentration index; Standard errors JEL classification: $\mathrm{I} 112$

\section{Introduction}

This paper clarifies the relationship between two widely used indices of health inequality, namely the relative index of inequality $(R I I)$ and the concentration index (CI). It explains why these are superior to other indices used in the empirical literature. The paper also clarifies the role that demographic standardization plays in the analysis of socioeconomic inequities in health.

Since the indices of health inequality are generally estimated from sample observations, it is useful to be able to test whether any observed differences in

\footnotetext{
* Corresponding author.
}

This paper derives from the project Equity in the finance and delivery of health care in Europe'. which is funded by the European Community's Biomet Programme (contract BMH1-C T92-608). We are grateful to the EC for financial support. We are also grateful to Han Bliechrodt for his help in the research leading up to this paper and for him. Alok Bhargava and three referees for helpful comments on a previous draft. We alone are responsibie for any errors. 
their values are statistically significant. This paper develops accurate distribution-free asymptotic estimators of the standard errors of both the RII and CI. There is, of course, an extensive literature on the sampling properties of the Gini index to which the CI is related (Nygord and Sanstöm, 1981; Kakwani, 1990; Cowell, 1989). These sampling distributions are derived by applying Hoeffding's (1948) theorem on order statistics. This methodology cannot be applied to derive the sampling distribution of CIs because they can be both negative and positive and, therefore, cannot be written in the form of orcier statistics. Thus, the derivations of the standard error formulae of this paper (presented in the Appendix) are new, providing more general results. The formulae in the current paper reduce to the standard formulae for the Gini index when ranking by ill-health coincides with socioeconomic ranking (see Appendix B).

\section{Two indices of socioeconomic inequality in health}

The illness concentration curve plots the cumulative proportion of the population - ranked by socioeconomic status (SES), beginning with the least advantaged - against the cumulative proportion of illness. ${ }^{1}$ If $L(s)$ lies above (below) the diagonal, inequalities in illness favour the more (less) advantaged members of society. Health inequality can be measured by the $C I$, denoted below by $C$, defined as twice the area between $L(s)$ and the diagonal:

$$
C=1-2 \int_{0}^{1} L(s) \mathrm{d} s .
$$

$C$ takes a value of zero when $L(s)$ coincides with the diagonal and is negative (positive) when $L(s)$ lies above (below) the diagonal. ${ }^{2}$

On individual-level data $C$ can be computed straightforwardly. Let $x_{i}$ $(i=1, \ldots, n)$ be the ill-health score of the $i$ th individual. Each of the $n$ individuals are then ranked according to their SES, beginning with the most disadvantaged. $C$ can then be calculated as

$$
C=\frac{2}{n \cdot \mu} \sum_{i=1}^{n} x_{i} R_{i}-1
$$

\footnotetext{
${ }^{\prime} \mathrm{Cf}$. Wagstatf et al. (1989). On concentration curves and indices more generally, see Kak wani (1977. 1980) and Lambert (1993).

'The minimum and maximum values of $C$ using individual-level data are -1 and +1 respectively: these occur when all the population's ill-health is concentrated in the hands of the most disadvanlages person and the least disadvantages person respectively.
} 
where $\mu=(1 / n) \sum_{i=1}^{n} x_{i}$ is the mean level of ill-health and $R_{i}$ is the relative rank of the ith person. Eq. (2) make clear the dependence of $C$ on the socioeconomic dimension to the distribution of ill-health. Suppose that person i's ill-health falls by an amount $\Delta$, whilst person $j$ 's rises by the same amount. The effect on $C$ is given by

$$
\Delta C=\frac{2}{n \cdot \mu} \Delta\left(R_{i}-R_{j}\right)
$$

which is clearly positive (negative) if $i$ is more (less) disadvantaged than $j$. This sensitivity to the socioeconomic dimension to inequalities in health is not a feature of several other indices used in the literature. ${ }^{3}$ Eq. (2) also makes clear that $C$ depends on the ill-health of all members of society.

Inequalities in health are frequently investigated using grouped data, the groups comprising socioeconomic groups (SEGs), social classes, groups of persons with similar levels of educational attainment, or income groups. ${ }^{4}$ Let $\mu_{t}$ $(t=1, \ldots, T)$ be the morbidity rate of the $t$ th SEG and $f_{t}$ its population share. Rank the $T$ SEGs according to their SES, beginning with the most disadvantaged. If $L(s)$ is assumed to be piecew: linear, $C$ can be calculated as

$$
C=\frac{2}{\mu} \sum_{t-1}^{T} f_{t} \mu_{t} R_{t}-1
$$

$\sum_{t=1}^{T} f_{t} \mu_{t}$ being the mean morbidity rate, and $R_{t}$ is the relative rank of the $t$ th SEG, defined as

$$
R_{t}=\sum_{i=1}^{i-1} f_{i}+\frac{1}{2} f_{l}
$$

and indicating the cumulative proportion of the population up to the midpoint of each group interval. ${ }^{5}$

\footnotetext{
${ }^{3}$ It is not true of the Gini coefficient (cf., e.g., Illsley and Le Grand, 1986), the index of dissimilarity (cf., e.g., Preston et al., 1981), or the index of inequality (cf. Pappas et al., 1993). On this point cf. Wagstafl et al. (1991).

${ }^{4}$ In cross-country comparisons in particular, the scope for meaningfull comparison is often limited by diflerence in the way groups are defined. Some researchers have succeeded, however, in achieving a higher degree of comparability with existing surveys (see, e.g. Vagerö and Lundberg, 1989: Kunst and Mackenbach, 1994). In any case the problem is likely to become smaller over time, since various international organisations have launched initiatives aimed at harmonizing survey questions relevant to this area of research.

${ }^{5} \mathrm{Eq}$. (3) makes clear the fact that, where grouped data are used, $C$ depends on the relative sizes of the SFGs, a property not shared by the range.
} 
The RII, which has invariably been used in the context of grouped data, is the slope of a regression of a group's relative morbidity, $\mu_{t} / \mu$, on its relative rank, $R_{t}$. The grouped nature of the data calls for Weighted Least Squares (WLS), the WLS estimate of the RII being easily obtained by running OLS on

$$
\left[\mu_{t} / \mu\right] \sqrt{f_{t}}=\alpha \cdot \sqrt{f_{t}}+\beta \cdot R_{t} \sqrt{f_{t}}+u_{t},
$$

the estimator of $\hat{\beta}$, of which is equal to the RII. The RII and the CI are, in fact, related to one another by (cf. Wagstaff et al., 1991)

$$
\hat{\beta}=C / 2 \sigma_{R}^{2},
$$

where $\sigma_{R}^{2}=\sum_{t=1}^{T} f_{t}\left(R_{t}-\frac{1}{2}\right)$.

\section{Demographic factors and avoidable inequality}

So far we have said nothing about the role demographic factors in generating health inequality - illness rates have been assumed to be crude illness rates. Comparing $L(s)$ with the diagonal, or the RII with zero, presupposes that all socioeconomic inequalities in illness are avoidable. This is unrealistic, since there are biological influences on health that are to a large degree unalterable. It is clearly unreasonable, for example, to suppose that a person of 85 could be made as healthy as a 20 -year old. The diagonal is thus an unsuitable benchmark against which to compare $L(s)$ and zero is an unsuitable benchmark against which to compare the RII if crude morbidity rates are used in its calculation.

One approach is to use the direct method of standardization. This requires that grouped data be used and involves applying the age -sex-specific average illness rates of each SEG to the age and gender structure of the population (cf., e.g., Rothman, 1986)]. The standardized illiess rate for SEG $\iota$ is equal to

$$
\mu_{t}^{+}=\sum_{d} n_{d} \mu_{d i} / n
$$

where $n_{d}$ is the number of persons in the dth demographic group in the population as a whole. $\mu_{d t}$ is the morbidity rate amongst persons in the $d$ th demographic group in SEG $t$. If age-sex-specific morbidity rates are equal to the population rates in each age-sex group (i.e., $\mu_{d t}=\mu_{d} \forall d$ ), the standardized rates will not vary across SEGs (i.e., $\mu_{t}^{+}=\mu \forall t$ ). The extent of avoidable inequality could be assessed by means of the RII with the directly standardized rates (i.e., the $\mu_{t}^{+}$) being used instead of the unstandardized rates (i.e., the $\mu_{t}$ ). Alternatively a directly standardized concentration curve can be constructed. denoted by $L^{+}(s)$, based on the each of the T SEGs' shares of standardized illness:

$$
S_{t}^{+}=f_{t} \sum_{d} n_{d} \mu_{d l} / \sum_{d} n_{d} \mu_{d} ;
$$


these are equal to $f_{t}$ if $\mu_{d t}=\mu_{d} \forall d$. If $L^{+}(s)$ lies above the diagonal, the less advantages SEGs experience higher age-sex-specific illness rates than the population as a whole, whilst the opposite is true if $L^{+}(s)$ lies below the diagonal. Thus an alternative measure of avoidable inequalities in health is thus twice the area between $L^{+}(s)$ and the diagonal:

$$
C^{+}=1-2 \int_{0}^{1} L^{+}(s) \mathrm{d} s
$$

which is negative (positive) if avoidable inequalities favour the more (less) advantages SEGs and zero if there are no avoidable inequalities in health.

The fact that the direct standardization requires the use of grouped data is a disadvantage, since the number of SEGs used will affect the numerical values of $C$ and $C^{+}$. An alternative is to use the indirect method of standardization, which can also be used on individual-level data. This involves replacing person i's degree of illness by the degree of illness suffered on average by persons of the same age and gender as person $i$ (cf., e.g., Rothman, op. cit.). Let $L^{*}(s)$ be the corresponding concentration curve. If the more disadvantages members of society are in the demographic groups that are most prone to illness, $L^{*}(s)$ will lie above the diagonal, indicating that it is unreasonable to suppose that $L(s)$ could ever be brought down as far as the diagonal. If, by contrast, the more disadvantaged members of society are in those demographic groups that are least prone to illness, $L^{*}(s)$ will lie below the diagonal, indicating that it would be feasible to bring $L(s)$ below the diagonal. An alternative measure of avoidable inequalities in health is thus twice the area between $L(s)$ and $L^{*}(s)$ :

$$
I^{*}=2 \int_{0}^{1}\left[L^{*}(s)-L(s)\right] \mathrm{d} s=C-C^{*},
$$

which is negative ( positive) if there are avoidable inequalities favouring the more (less) advantaged members of society. $C^{*}$ can be computed straightforwardly using Eq. (2) but replacing the actual illness score 'vith the indirectly standardized score.

\section{Statistical inference}

Consider first the case of grouped data. Application of OLS to Eq. (5) automatically provides a standard error for the RII. ${ }^{6} \mathrm{~A}$ standard error for $C$ can

\footnotetext{
"This is, in effect. the method used by Kunst and Mackenbach (1994).
} 
easily be obtained from the following convenient regression:

$$
2 \sigma_{R}^{2}\left[\mu_{t} / \mu\right] \sqrt{n_{t}}=x_{1} \cdot \sqrt{n_{t}}+\beta_{1} \cdot R_{t} \sqrt{n_{t}}+u_{t} .
$$

The OLS etimator $\beta_{1}$ is equal to

$$
\hat{\beta}_{1}=\frac{2}{\mu} \sum_{t=1}^{T} f_{t}\left(\mu_{t}-\mu\right)\left(R_{t}-\frac{1}{2}\right)
$$

which, from Eq. (3), shows that $\hat{\beta}_{1}$ is equal to $C$. These standard errors are not, however, wholly accurate, since the observations in each regression equation are not independent of one another. In the Appendix we develop the following estimators which take into account the serial correlation in the data:

$$
\begin{aligned}
& \operatorname{var}(\hat{\beta})=\frac{1}{n \sigma_{R}^{4}}\left[\sum_{t=1}^{T} e_{t}^{2} f_{t}-\left(\sum_{t=1}^{T} e_{t} f_{t}\right)^{2}+\frac{1}{\mu^{2}} \sum_{t=1}^{T} \sigma_{t}^{2}\left(R_{t}-\frac{1}{2}-\frac{1}{2} C\right)^{2}\right], \\
& \operatorname{var}(\hat{C})=\frac{1}{n}\left[\sum_{t=1}^{T} f_{t} a_{t}^{2}-(1+C)^{2}\right]+\frac{1}{n \mu^{2}} \sum_{t-1}^{T} f_{t} \sigma_{t}^{2}\left(2 R_{t}-1-C\right)^{2},
\end{aligned}
$$

where $\sigma_{t}^{2}$ is the variance ill-health score in the $t$ th SEG,

$$
\begin{aligned}
& c_{t}=\frac{1}{2} a_{t}-\hat{\beta}\left[1+R_{t}^{2}-\left(s_{t}+s_{t-1}\right)\right], \\
& a_{t}=\frac{\mu_{t}}{\mu}\left(2 R_{t}-1-C\right)+2-q_{t-1}-a_{t}, \\
& a_{t}=\frac{1}{\mu} \sum_{i=1}^{1} \mu_{i} f_{i,}
\end{aligned}
$$

being the ordinate of $L(s)_{,} q_{0}=0$, and

$$
s_{t}=\sum_{i=1}^{1} f_{i} R_{i},
$$

with $s_{0}=0$. If one or other of the standardization methods is being used, one clearly needs to replace $\mu$ by the standardized value. If the direct method of standardization is being used, $\sigma_{t}^{2}$ is, by definition, equal to zero for all $T$ SEGs and $n$ in the denominator has to be replaced by $T$, the reason being that $\mu_{t}^{+}$is defined only at the level of the SEG, not at the level of the individual, and hence there are only $T$ independent observations. ${ }^{7}$

\footnotetext{
The estimation of a statistic from grouped data always give larger standard crrors because of loss of degrees of freedom. If $\sigma_{1}^{2}$ was not equal to zero, then there are $n$ degrees of freedom. But if we assume zero variation within groups, the degrees of freedom are reduced to $T$. the number of groups. Thus, the effective sample size is $T$ not $n$.
} 
On ungrouped data, the simplest way to obtain a standard error for $C$ is to run OLS on the following equation:

$$
2 \sigma_{R}^{2}\left[x_{i} / \mu\right]=x_{2}+\beta_{2} \cdot R_{i}+u_{i}
$$

The estimator of $\beta_{2}$ is equal to

$$
\hat{\beta}_{2}=\frac{2}{n \cdot \mu} \sum_{i=1}^{n}\left(x_{t}-\mu\right)\left(R_{i}-\frac{1}{2}\right),
$$

which, from Eq. (2), shows that $\hat{\beta}_{2}$ is equal to $C$. From Eq.(14) one can obtain a standard error for $C$. Using the same method, one can obtain a standard error for $C^{*}$, but a standard error for $I^{*}$ is not easily obtained because the sample estimates of $C$ and $C^{*}$ are not independently distributed. We can, however, estimate a standard error for $I^{*}$ by means of the following convenient regression:

$$
2 \sigma_{R}^{2}\left[\frac{x_{i}}{\mu}-\frac{x_{i}^{*}}{\mu^{*}}\right]=x_{3}+\beta_{3} \cdot R_{i}+u_{i},
$$

where $x_{i}^{*}$ is the indirectly standardized value of $x_{i}$ and $\mu^{*}$ is the mean of $x_{i}^{*}$. The OLS estimate of $\beta_{3}$ in Eq. (21) will be equal to $I^{*}$ and from this regression we also obtain a standard error for $I^{*}$. As in the case of grouped data, the standard errors obtained using these convenient regressions are not entirely accurate. More accurate standard errors are obtained by taking into account the serially correlated nature of the error structure. Bearing in mind that with individuallevel data the analogue of $\sigma_{t}^{2}$ is zero, the variance of $\hat{C}$ can be calculated as ${ }^{8}$

$$
\operatorname{var}(\hat{C})=\frac{1}{n}\left[\frac{1}{n} \sum_{i=1}^{n} a_{t}^{2}-(1+C)^{2}\right]
$$

where $a_{i}$ is defined along the lines of $a_{1}$. The variance of $\hat{I}^{*}$, by contrast. can be computed as

$$
\operatorname{var}\left(\hat{I}^{*}\right)=\frac{1}{n}\left[\frac{1}{n} \sum_{i=1}^{n}\left(a_{i}-a_{i}^{*}\right)^{2}-l^{* 2}\right],
$$

where $a_{t}^{*}$ is defined analogously to $a_{i}$ except that indirectly standardized values are used in its calculation instead of actual values.

\footnotetext{
${ }^{8}$ See Appendix $\mathbf{B}$ for the derivation of the result given in (22)
} 


\section{Empirical illustrations}

In this section we report some empirical illustrations using data from the combined 1980 and 1981 Dutch Health Interview Surveys (HIS). The sample size, after deletion of cases with missing information, is 10,232 persons. Our stratifying variable is pre-tax household income per equivalent adult. ${ }^{9} \mathrm{We}$ use two widely available indicators of ill-health - chronic illness (a dummy variable indicating the presence or absence of any chronic illness) and self-assessed health (the HIS question being 'How is your heaith in general?', to which respondents could reply 'good', 'fair', 'varies', and 'poor'). ${ }^{10}$ In the case of the former, we simply used the actual values of the variable. In the case of the latter, we have assumed that underlying the categorical self-assessed health variable is a continuous latent ill-health variable with a standard lognormal distribution (cf. Wagstaff and van Doorslaer, 1994a). ${ }^{11}$

For the purpose of illustrating the methods for grouped data, we have divided the sample into income deciles. In general, the lower deciles suffer somewhat higher levels of ill-health than the higher deciles (Table 1). With the exception of the bottom decile, the effect of the standardization is to reduce the mean ill-health levels of the lower income groups and raise those of the upper income groups. The negative values of $C, C^{+}, \beta$, and $\beta^{+}$in Table 2 imply that even after taking into account the demographic structure of the sample, inequalities in health favour the better-off. The values suggest that inequality is more pronounced if health is mensured by self-assessed health than if it is measured by chronic illness. ${ }^{2}$ The standardized variants of $C$ and $\beta\left(C^{+}\right.$and $\left.\beta^{+}\right)$are smaller than the unstandardized variants $(C$ and $\beta$ ), implying that some of the inequality in crude morbidity rales is unavoidable and due simply to the age structure of the sample. The standard errors for $C^{+}$and $\beta^{+}$are a good deal smaller than those of $C$ and $\beta$ - this presumably reflects the fact that the direct standardization reduces the variation in illness rates. The standard errors obtained using the two methods differ somewhat, but not apparently in any predictable way. It

\footnotetext{
"The equivalence scale used is that used by th. Dus? Central Burealu of Statistics (cf. Schiepers. 1988). The scale takes into account the number of aduits and children in the household, as well as the ige of the eldest child.

"Our methods could, of course, be applied with health measures other than our chosen indicators: see for instance Bhargava (1994).

"In effect, we obtain the values lor each of the four categories by dividing up the allea under the standard lognormal distribution according to stmple proportions falling into each of the four calcgorion

1: This a mclusion is consistent with other research where both self-inssessed health and chronic illness are meusured by dichotomous variables (cí. Kunst et al.. 1992: van Doorsliter. Wanstalf. and Rutten, 1993). The reason is probably that the chronic illness dicholomous variable insulliciently captures the underlving differences in the severity of chronic illness (O'Donnell and Propper. 1991).
} 
Table 1

Means for grouped data

\begin{tabular}{lllll}
\hline Decile & $\begin{array}{l}\text { Chronic } \\
\text { unstandardized }\end{array}$ & $\begin{array}{l}\text { Chronic } \\
\text { standardized }\end{array}$ & $\begin{array}{l}\text { SAH } \\
\text { unstandardized }\end{array}$ & $\begin{array}{l}\text { SAH } \\
\text { standardized }\end{array}$ \\
\hline 1 & 0.2808 & 0.2885 & 1.6317 & 1.6837 \\
2 & 0.3629 & 0.3125 & 1.9946 & 1.7684 \\
3 & 0.3405 & 0.3061 & 1.7503 & 1.6549 \\
4 & 0.2985 & 0.2844 & 1.6329 & 1.5769 \\
5 & 0.2965 & 0.2972 & 1.4760 & 1.4980 \\
6 & 0.2805 & 0.2944 & 1.3817 & 1.4341 \\
7 & 0.2622 & 0.2755 & 1.2733 & 1.3279 \\
8 & 0.2811 & 0.2967 & 1.3310 & 1.4285 \\
9 & 0.2600 & 0.2791 & 1.2468 & 1.2979 \\
10 & 0.2766 & 0.2870 & 1.2062 & 1.2544 \\
Total & 0.2940 & 0.2921 & 1.4925 & 1.4927 \\
\hline
\end{tabular}

Standardizations are direct standardizations and obtained using Eq. (8), with two gender groups and five age groups (18 -34. $35-44.55-64,65-74,75+)$.

Table 2

Concentration indices and standa: $d$ errors for grouped data

\begin{tabular}{|c|c|c|c|c|}
\hline & $\begin{array}{l}\text { Chronic } \\
\text { illnessi" }\end{array}$ & $\begin{array}{l}\text { Chronic } \\
\text { illness }{ }^{\mathrm{b}}\end{array}$ & $\mathrm{SAH}^{\mathrm{a}}$ & $\mathrm{SAH}^{\mathrm{b}}$ \\
\hline $\begin{array}{l}C \\
\operatorname{se}(C) \\
\text {-test: } C\end{array}$ & $\begin{array}{r}-0.0402 \\
0.0165 \\
-2.4332\end{array}$ & $\begin{array}{r}-0.0402 \\
0.0164 \\
-2.4512\end{array}$ & $\begin{array}{r}-0.0827 \\
0.0153 \\
-5.4164\end{array}$ & $\begin{array}{r}0.0827 \\
0.0139 \\
-5.9496\end{array}$ \\
\hline $\begin{array}{l}\mathrm{C}^{+} \\
\sec \left(\mathrm{C}^{+}\right) \\
\text {r-test: } \mathrm{C}^{+}\end{array}$ & $\begin{array}{r}0.0111 \\
0.2965 \\
-1.6912\end{array}$ & $\begin{array}{r}-0.0111 \\
0.2972 \\
-1.6838\end{array}$ & $\begin{array}{r}-0.0609 \\
0.0067 \\
-9.0509\end{array}$ & $\begin{array}{r}-0.0609 \\
0.0085 \\
-7.1824\end{array}$ \\
\hline $\begin{array}{l}\beta \\
\operatorname{se}(\beta) \\
t \text {-test: } \beta\end{array}$ & $\begin{array}{r}-0.2435 \\
0.1001 \\
-2.4332\end{array}$ & $\begin{array}{r}-0.2435 \\
0.0994 \\
-2.4478\end{array}$ & $\begin{array}{r}-0.5010 \\
0.0925 \\
-5.4164\end{array}$ & $\begin{array}{r}-0.5010 \\
0.0845 \\
-5.9297\end{array}$ \\
\hline $\begin{array}{l}\beta^{+} \\
\operatorname{se}\left(\beta^{+}\right) \\
t-\operatorname{test}: \beta^{+}\end{array}$ & $\begin{array}{r}-0.0672 \\
0.0397 \\
-1.6912\end{array}$ & $\begin{array}{r}-0.0672 \\
0.0352 \\
-1.9079\end{array}$ & $\begin{array}{r}-0.3690 \\
0.0408 \\
-9.0509\end{array}$ & $\begin{array}{r}-0.3690 \\
0.0513 \\
-7.1903\end{array}$ \\
\hline
\end{tabular}

$\sigma_{R}^{2}=0.0825$ in our sample.

"Obtained using convenient regressions (11) and (5).

"Obtained using formulae in Eqs. (3). (13). and (14).

is not possible to conclude, for example, either from the empirical illustrations or from the relevant formulae whether the method that corrects for serial correlation is likely to produce smaller or larger standard errors than the method that 
Table 3

Concentration indices and standard errors for individual-level data

\begin{tabular}{llrrr} 
& $\begin{array}{l}\text { Chronic } \\
\text { illness }\end{array}$ & \multicolumn{1}{l}{$\begin{array}{l}\text { Chronic } \\
\text { illness }\end{array}$} & \multicolumn{1}{c}{ SAII $^{\mathrm{a}}$} & \multicolumn{1}{c}{ SAII $^{\mathrm{b}}$} \\
\hline$C$ & -0.0404 & -0.0403 & -0.0837 & -0.0836 \\
$\operatorname{se}(C)$ & 0.0088 & 0.0096 & 0.0071 & 0.0086 \\
$t$-test: $C$ & -4.5764 & -4.2064 & -11.7778 & -9.6848 \\
$C^{*}$ & -0.0306 & -0.0305 & -0.0263 & -0.0262 \\
se $\left(C^{*}\right)$ & 0.0023 & 0.0055 & 0.0018 & 0.0057 \\
$i$-test: $C^{*}$ & -13.2148 & -5.5027 & -14.3930 & -4.6306 \\
$I^{*}$ & -0.0098 & -0.0099 & -0.0572 & -0.0574 \\
se $\left(I^{*}\right)$ & 0.0085 & 0.0086 & 0.0069 & 0.0068 \\
$t-$ test: $I^{*}$ & -1.1554 & -1.1480 & -8.3298 & -8.4962 \\
\hline
\end{tabular}

The $t$-ritios presented in the table were calculated from the coefficients and the standard errors to seven decimal places rather than the four in the table - hence the fact that calculating the $t$-ratios from the figures in the table give slightly different numbers.

"Obtained using convenient regressions regressions (19) and (21).

${ }^{b}$ Obtained using formulae in (2), (10), (22), and (23).

does not. In Table 2 the differences are fairly small and the $t$-values are such that using either method leads to the same conclusions concerning the existence of otherwise of significant inequalities in health. ${ }^{13}$

The indirect standardizations in Table 3 used in computing $C^{*}$ were obtained using regression analysis along the lines set out in van Vliet and van de Ven (1985) and Wagstaff and van Doorslaer (1994b). A logit model was used in the case of chronic illness, and OLS in the case of the self-assessed health variable, having first replaced the four ordinal scores with the latent ill-health variable scores. The standardized values, $x_{i}^{*}$, are then simply the predicted values retained from these regressions. The values of $C$ for chronic illness and selfassessed health in Table 3 are slightly smaller (i.e., larger in absolute size) than those obtained using the grouped data in Table 2. The standard errors of $C$ in Table 3, obtained using individual-level data, are substantially smaller (40-50\%) than those in Table 2, obtained using grouped data. The standard errors of $I^{*}$, by contrast, are not markedly different from those of $I^{+}$(i.e., $\mathrm{C}^{+}$) reported in Table 2 - this reflects the reduction in sample variation caused by the standardizations. The $t$-tests testing the significance of $I^{*}$ from zero suggest that there are

\footnotetext{
${ }^{13}$ The insignificance of inecpualities in standardized chronic illness rates may be due to the fact the chron illness questions in the HIS are posed in such a way as to encourage the reporting of even fairly 'minor' chronic conditions such as excema and migraine. which tend to be commonest among the higher income groups in the Netherlands (cf.. e.g., van Doorslaer. Wagstaff. and Janssen. 1993).
} 
no significant avoidable inequalities in the presence of chronic illness but that there are significant avoidable inequalities in self-assessed health. These are the same conclusions that were reached with the grouped data in Section 4, though the $t$-values for chronic illness are a good deal smaller in the case of $I^{*}$ and $I^{+}$.

\section{Conclusions}

We have shown that the RII and CI are closely related to one another and that it is possible to construct a variant of the $\mathrm{CI}$ to allow inequalities in health to be measured on individual-level data even with age-sex standardization. This allows the extra precision allowed for by individual-level data to be retained but at the same time allows one to net out of one's calculations the unavoidable component of health inequality attributable to the demographic structure of the sample. We have also derived asymptotic distribution-free standard errors for both the RII and the CI. Finally, the empirical illustrations in the paper suggest that there may, in practice, only be small differences between the standard errors obtained using the method which does not take into account serial correlation and those obtained using the method proposed in this paper which does take it into account. Surprisingly, the results also suggest that in this context the gain in precision associated with the use of individual-level data may not always be that large.

\section{Appendix A: Derivation of standard errors based on groupc data}

Suppose there are $T$ socioeconomic groups and $f$, is the population relative frequency of the $t$ th group, then $\sum_{t=1}^{T} f_{t}=1$. It is reasonable to assume that the sample absolute frequencies $\left(n_{1}, n_{2}, \ldots, n_{t}\right)$ based on a sample of $n$ individuals follow a multinomial distribution so that the means, variances, and covariances of the sample estimates of the relative frequencies are given by

$$
\mathrm{E}\left(\hat{f}_{t}\right)=f_{t}, \quad \operatorname{var}\left(\hat{f}_{t}\right)=\frac{1}{n} f_{t}\left(1-f_{t}\right), \quad \operatorname{cov}\left(f_{t}, f_{t^{\prime}}\right)=-\frac{1}{n} f_{t} f_{t^{\prime}}, \quad \text { if } \quad t \neq t^{\prime},
$$

where $t=1,2, \ldots, T$. Then one can always write

$$
\hat{f}=f+s,
$$

where $f^{\prime}=\left(f_{1}, f_{2}, \ldots, f_{T}\right), \hat{f}=\left(\hat{f}_{1}, \hat{f}_{2}, \ldots, \hat{f}_{T}\right)$, and $\varepsilon^{\prime}=\left(\varepsilon_{1}, \varepsilon_{2}, \ldots, \delta_{T}\right), \varepsilon$ being the error vector such that

$$
\mathrm{E}(s)=0 \text { and } \mathrm{E}\left(c x^{\prime}\right)=\frac{1}{n} \Sigma \text {, }
$$


$\Sigma$ being the $T \times T$ matrix given by

$$
\Sigma=\operatorname{diag}(f)-f f^{\prime} .
$$

Let $m^{\prime}=\left(\mu_{1}, \mu_{2}, \ldots, \mu_{T}\right), \mu_{t}$ being the average morbidity of the $t$ th SEG, then the mean morbidity of the society is given by

$$
\mu=m^{\prime} f
$$

Let $\hat{\mu}_{t}$ be the sample estimate of $\mu_{t}$, then one can always write

$$
\hat{\mu}_{t}=\mu_{t}+\delta_{t},
$$

where $\delta_{\mathrm{t}}$ is the error term such that

$$
\mathrm{E}\left(\delta_{t}\right)=0 \text { and } \mathrm{E}\left(\delta_{t}^{2}\right)=\sigma_{t}^{2} / n_{t} .
$$

If $\hat{m}^{\prime}=\left(\hat{\mu}_{1}, \hat{\mu}_{2}, \ldots, \hat{\mu}_{T}\right)$ and $\delta^{\prime}=\left(\delta_{1}, \delta_{2}, \ldots, \delta_{T}\right)$, then one can write

$$
\hat{m}=m+\delta,
$$

such that $\mathrm{E}(\delta)=0$ and $\mathrm{E}\left(\delta \delta^{\prime}\right)=(1 / n) \Omega$, where $\Omega=\operatorname{diag}\left(\sigma^{2}\right)[\operatorname{diag}(f)]^{-1}, \sigma^{2}$ being the $T \times 1$ vector with the $t$ th element equal to $\sigma_{t}^{2}$.

A sample estimate of the mean morbidity rate of the society is given by

$$
\hat{\mu}=\hat{m}^{\prime} \hat{f}
$$

which in view of (A.1) and (A.5) can be written as

$$
\hat{\mu}=\mu+m^{\prime} x+f^{\prime} \hat{\delta},
$$

which on assuming that $\varepsilon$ and $\delta$ are independent gives the result: $\sqrt{n}(\hat{\mu}-\mu)$ follows normal distribution with mean zero and variance $\left(m^{\prime} \Sigma m+f^{\prime} \Omega f\right)$. This gives the variance of $\hat{\mu}$ as

$$
\operatorname{var}(\hat{\mu})=\frac{1}{n}\left[\sum_{t=1}^{T} f_{i}\left(\mu_{t}-\mu\right)^{2}+\sum_{i=1}^{T} f_{t} \sigma_{t}^{2}\right] .
$$

Let us now introduce a $T \times T$ matrix

$$
A=\left[\begin{array}{ccccc}
\frac{1}{2} & 0 & 0 & \ldots & 0 \\
1 & \frac{1}{2} & 0 & \ldots & 0 \\
1 & 1 & \frac{1}{2} & \ldots & 0 \\
1 & 1 & 1 & \ldots & \frac{1}{2}
\end{array}\right] .
$$


which has the property that

$$
A+A^{\prime}=\iota^{\prime}
$$

where $t^{\prime}=(1,1, \ldots, 1)$ is the row vector of $T$ elements, each of which being equal to 1 .

Let $R^{\prime}=\left(R_{1}, R_{2}, \ldots, R_{r}\right), R_{1}$ being the relative rank of the $t$ th SEG (defined in (4)); then $R=A f$. A sample estimate of $R$ will then be given by $\hat{R}=A \hat{f}$ which from (A.1) gives

$$
\hat{R}=R+A \varepsilon
$$

We may now write the sample estimate of the concentration measure $C$ as

$$
\hat{C}=\frac{2}{\hat{\mu}} \hat{m}^{\prime}[\operatorname{diag}(\hat{f})] \hat{R}-1 .
$$

Substituting (A.1), (A.5), and (A.8) into (A.9) and applying Taylor's expansion gives

$$
\hat{C}=C+a^{\prime} \varepsilon+b^{\prime} \dot{\delta}+\mathrm{O}_{p}\left(\frac{1}{n}\right)
$$

where

$$
\begin{aligned}
& a=\frac{1}{\mu}\left[2 \operatorname{diag}(R) m+2 A^{\prime} \operatorname{diag}(f) m-(1+C) m\right] \\
& b=\frac{1}{\mu}[2 \operatorname{diag}(f) R-(1+C) f],
\end{aligned}
$$

and $O_{p}(1 / n)$ includes all the remainder terms that are of order $1 / n$ or less in probability. Eq. (A.10) immediately gives the result: $\sqrt{n}(\hat{C}-C)$ follows an asymptotic normal distribution with zero mean and variance $\left(a^{\prime} \Sigma a+b^{\prime} \Omega b\right)$ which gives

$$
\operatorname{var}(\hat{C})=\frac{1}{n}\left[\sum_{t=1}^{T} f_{i} a_{t}^{2}-(1+C)^{2}\right]+\frac{1}{n \mu^{2}} \sum_{t=1}^{T} f_{t} \sigma_{t}^{2}\left(2 R_{t}-1-C\right)^{2}
$$

where

$$
a_{t}=\frac{\mu_{t}}{\mu}\left(2 R_{t}-1-C\right)+2-q_{t-1}-q_{t} \text { and } q_{t}=\frac{1}{\mu} \sum_{r=1}^{t} \mu_{r} f_{r} .
$$

being the ordinate of the concentration curve. 
The relative index of inequality $\mathrm{RII}$, which we denote by $\beta$, is related to the concentration as $\beta=C / 2 \sigma_{R}^{2}$, where $\sigma_{R}^{2}=\sum_{t=1}^{T} f_{t}\left(R_{t}-\frac{1}{2}\right)^{2}$ (Eq. (6)). A sample estimate of $\beta$ is given by

$$
\hat{\beta}=\hat{C} / 2 \hat{\sigma}_{R}^{2},
$$

where

$$
\hat{\sigma}_{R}^{2}=\sum_{t=1}^{T} \hat{f}_{t}\left(\hat{R}_{t}-\frac{1}{2}\right)^{2}
$$

Utilizing (A.8) and (A.101 into (A.13) and Taylor's expansion gave

$$
\operatorname{var}(\hat{\beta})=\frac{1}{n \sigma_{R}^{4}}\left[\sum_{t=1}^{T} f_{t} e_{t}^{2}-\left(\sum_{t=1}^{T} f_{t} e_{t}\right)^{2}+\frac{1}{\mu^{2}} \sum_{t=1}^{T} \sigma_{t}^{2}\left(R_{t}-\frac{1}{2}-\frac{C}{2}\right)^{2}\right]
$$

where

$$
e_{t}=\frac{a_{t}}{2}-\hat{\beta}\left[1+R_{t}^{2}-s_{t}-s_{t-1}\right], \quad s_{t}=\sum_{r=1}^{t} f_{r} R_{r}, \quad s_{0}=0 .
$$

If we assume that the groups are homogeneous (which is the case when individual observations are available), $\sigma_{t}^{2}$ will be zero in each group. The formulae of $\operatorname{var}(\hat{C})$ and $\operatorname{var}(\hat{\beta})$ will simplify considerably. In this situation, $C$ given in (A.10) will be given by

$$
\hat{C}=C+a^{\prime} t+O_{p}\left(\frac{1}{n}\right)
$$

Similarly, if the sample estimate of standardized $C^{*}$ can be written as

$$
\hat{C}^{*}=C^{*}+a^{*} \varepsilon+\mathrm{O}_{p}\left(\frac{1}{n}\right)
$$

where the $t$ th element of $a^{*}$ is given by

$$
a_{t}^{*}=\frac{\mu_{t}^{+}}{\mu^{+}}\left(2 R_{t}-1-C^{*}\right)+2-q_{t}^{*}-q_{t-1}^{*},
$$

where $\mu_{t}^{+}$and $\mu^{+}$are the standardized morbidity means and $q_{t}^{*}=$ $\left(1 / \mu^{+}\right) \sum_{r=1}^{t} \mu_{r}^{+} f_{r}$ is the ordinate of the concentration curve $L^{*}(s)$.

A sample estimate of $I^{*}$ will then be given by

$$
\hat{I}^{*}=\hat{C}-\hat{C}^{*},
$$


which in view of (A.15) and (A.16) becomes

$$
\hat{I}^{*}=I^{*}+\left(a-a^{*}\right)^{*} \varepsilon+\mathrm{O}_{p}\left(\frac{1}{n}\right)
$$

This immediately lead to the result that $\sqrt{n}\left(\hat{I}^{*}-I^{*}\right)$ follows an asymptotic normal distribution with variance equal to $\left(a-a^{*}\right) \Sigma\left(a-a^{*}\right)$, which gives

$$
\operatorname{var}\left(\hat{I}^{*}\right)=\frac{1}{n}\left[\sum_{t=1}^{T}\left(a_{t}-a_{t}^{*}\right) f_{t}-I^{* 2}\right]
$$

\section{Appendix B: Derivation of standard errors based on individual observations}

Eq. (A.11) provides the standard errors of $\hat{C}$ computed on the basis of grouped data. We may now give an alternative derivation of the variance of $\tilde{C}$ estimated on the basis of individual observations. $\bar{C}$ can be written as

$$
\tilde{C}=\frac{2}{n \hat{\mu}} \sum_{i=1}^{n} x_{i} \tilde{R}_{i}-1,
$$

where $x_{i}$ is the ill-health score of the $i$ th individual and $\tilde{R}_{i}=(2 i-1) / 2 n$, where $i$ is the $i$ th rank when individuals in the sample are arranged in ascending order of $x_{i}$. It will be useful to write (B.1) as

$$
\tilde{c}=\hat{d} / 2 \hat{\mu},
$$

where

$$
\begin{aligned}
& \hat{d}=\frac{1}{n} \sum_{i=1}^{n} \hat{d}_{i}, \\
& \hat{d}_{i}=2 x_{i} \tilde{R}_{i}-\hat{\mu}\left(\hat{q}_{i}+\hat{q}_{i-1}\right)-\left(x_{i}-\hat{\mu}\right), \\
& \hat{q}_{i}=\sum_{j=1}^{i} x_{j} / \sum_{j=1}^{n} x_{i} .
\end{aligned}
$$

Then following Fraser (1957), we obtain

$$
\begin{aligned}
& n \operatorname{vâr}(\hat{\mu})=\frac{1}{n} \sum_{i=1}^{n}\left(x_{i}-\hat{\mu}\right)^{2}, \\
& n \operatorname{vâr}(\hat{d})=\frac{4}{n} \sum_{i=1}^{n}\left(d_{i}-\hat{d}\right)^{2}, \\
& n \operatorname{cov}(\hat{d}, \hat{\mu})=\frac{2}{n} \sum_{i=1}^{n}\left(x_{i}-\hat{\mu}\right)\left(d_{i}-\lambda\right) .
\end{aligned}
$$


Then applying the $\delta$ method given in Rao (1965. p. 321) the estimated variance of $\tilde{C}$ from (B.2) is obtained as

$$
\begin{aligned}
\operatorname{vâr}(\tilde{C}) & =\frac{1}{4 n \hat{\mu}}\left[\operatorname{vâr}(\hat{d})+4 \tilde{C}^{2} \operatorname{vâr}(\hat{\mu})-4 \tilde{C} \operatorname{côv}(\hat{d}, \hat{\mu})\right] \\
& =\frac{1}{4 \hat{\mu}^{2} n} \sum_{i=1}^{n}\left(\hat{d}_{i}-\hat{d}-\tilde{C} x_{i}+\tilde{C} x_{i}+\tilde{C} \hat{\mu}\right)^{2} .
\end{aligned}
$$

Note that one can also derive $\operatorname{vâr}(\tilde{C})$ from Eq. (A.11) by substituting $\sigma_{t}^{2}=0$ which gives

$$
\operatorname{vâr}(\tilde{C})=\frac{1}{n} \sum_{i=1}^{n}\left(a_{i}-1-\tilde{C}\right),
$$

where

$$
\hat{a}_{i}=\frac{x_{i}}{\hat{\mu}}\left(2 \hat{R}_{i}-1-\tilde{C}\right)+2-\hat{q}_{i}-\hat{q}_{i-1} .
$$

Comparing (B.3) and (B.4) we note that the two expressions are identical. Thus, the two alternative derivation of the standard errors give identical results.

\section{References}

Bhargava, Alok, 1994, Modelling the health of Filipino chindren. Journal of the Royal Statistical Sociely A 157. Part 3.

Cowell, F.A., 1989. Sampling varimes and decomposable inecguality measures, Journal of Economelrics $42,27-41$.

Doorslaer, E, van. A. Wagitilf, and F, Rutten, eds., 1993a, Equity in the finance and delivery of health care: An international prospective (Oxford University Press, Oxford).

Doorslaer, E. van, A. Wagstaff. and R. Janssen. 1993b. Equity in the finance and delivery of health care in the Netherlands, in: E. van Doorslaer, A. Wagstaff, and F. Rutten. eds., Equity in the finunce and delivery of health care: An international perspective (Oxford University Press, Oxford).

Fraser, 1957, Non-parametric methods in statistics (New York, NY).

Hoeffding. W., 1948. A class of statistics with asymptotic normal distribution. Annals of Mathematical Statistics XIX, $293 \cdots 325$.

Illsley, R. and J. Le Grand, 1987, The measurement of inequality in health, in: A. Williams, ed., Health and economics (Macmillan, London).

Jenkins, S., 1986. Calculating income distribution indices from microdata, Nation:: Tax Journal 61. $139-142$.

Kakwani. N.C.. 1977, Mensurement of tax progessivity: An international comparison, Economic Journal 87, 7180.

Ke'kwani, N.C.. 1980. Income inequality and poverty: Methods of estimation and policy application (Oxford University Press, New York, NY).

Kakwani, N.C.. 1990, Large sample distributions of severalf inequality measures: With application to Côte d'Ivoire, in: R.A.L. Carter. J. Dutta, and A. Ullah, eds.. Contributions to econometric theory and application (Springer-Verlag. New York, NY). 
Kunst, A.E. and J.P. Mackenbach, 1994, Size of mortality differences associated with educational level in nine industrialized countries. American Journal of Public Health 84, 932-937.

Kunst, A.E., J. Geurts, and L. van den Berg, 1992, International variation in socioeconomic mequalities in self-reported health (Dutch Central Bureati of Statistics, Voorburg).

Lambert, P.J., 1993, The distribution and redistribution of income: A mathematical analysis (Manchester University Press, Manchester).

Lerman. R.I. and S. Yitzhaki, 1984, A note on the calculation and interpretation of the Gini index, Economics Letters 15, 363-368.

Nygärd, F. and A. Sandström, 1981, Measuring income inequality (Almqvist \& Wiksell International, Stockholm).

O'Donnell, O. and C. Propper, 1991, Equity in the distribution of UK National Health Service resources, Journal of Health Economics 10, 1-19.

Pappas, G., S. Queen, W. Hadden, and G. Fisher, 1993, The increasing disparity in mortality between socioeconomic groups in the United States, 1960 and 1986, New England Journal of Medicine 329, 103-108.

Preston, S.H., M.R. Haines, and E. Pamuk, 1981, Effects of industrialization and urbanization on mortality in developed countries, in: Solicited papers, Vol. 2, IUSSP 19th international population conference (IUSSP, Liege).

Rao, C.R., 1965, Linear statistical inference and its applications (Wiley, New York, NY).

Rothman, K., 1986, Modern epidemiology (Little, Brown \& Co., Boston, MA).

Schiepers, J., 1988, Huishoudensequivalententiefactoren volgens de budgetverdelingsmethode, Sociaal-economische Maandstatistiek Suppl. 7, 28-37.

Vägerö, D. and O. Lundberg, 1989, Health inequalitics in Britain and Sweden, The Lancet II, 35- 36.

Vliet, R. van and W. van de Ven, 1985, Differences in medical consumption between publicly and privately insured in the Netherlands: Standardization by means of multiple regression. Paper presented to international meeting on health economics (Applied Econometrics Association, Rotterdam).

Wagstaff, A. and F. van Doorslaer, 1994a, Measuring inegualities in health ia the presence of multiple-category morbidity indicators, Health Economics 3, 281 291.

Wagstalf, A. and E. van Doorslier, 1994b, A new approach to the measurement of eguity in the delivery of health care, Equity project working paper no. 6 (Erasmus University, Rotterdam).

Wagstafr, A.. E. van Doorslace, and P. Paci. 1989, Equity in the finance and delivery of health care: Some tentative cross-country compatisons, Oxford Review of Economic Policy 5. 89 112.

Wagstaff, A., P. Paci, and E. van Doorslaer, 1991, On the measurement of inequalities in health, Social Science and Medicine 33, 545-557. 